\title{
Differential effect of vitamin D on NOD2- and TLR-induced cytokines in Crohn's disease
}

\author{
S Dionne ${ }^{1}$, MR Calderon ${ }^{2}$, JH White ${ }^{2}$, B Memari ${ }^{2}$, I Elimrani ${ }^{1}$, B Adelson ${ }^{1}$, C Piccirillo $^{1}$ and EG Seidman $^{1}$
}

Accumulating evidence implicates defective innate immunity in the pathogenesis of Crohn's disease (CD). Ineffectual NOD2 (nucleotide-binding oligomerization domain 2) is the most common susceptibility gene contributing to CD. Vitamin $\mathrm{D}(\mathrm{vD})$, a potent modulator of innate and adaptive immunity, induces NOD2 gene expression and its downstream function. We hypothesized that the hormonal form of $\mathrm{vD}(1,25 \mathrm{D})$ could beneficially modulate innate immune function in $\mathrm{CD}$. Using peripheral mononuclear cells and monocyte-derived dendritic cells (Mo-DCs) from CD, it was found that 1,25D decreased Toll-like receptor (TLR)-induced cytokine production and enhanced cytokine levels induced by muramyl dipeptide (MDP), the NOD2 ligand. 1,25D increased the synergistic effect provided by NOD2 and TLR co-activation on interleukin (IL)-10, IL-23, and tumor necrosis factor-alpha (TNF- $\alpha$ ). Whereas 1,25D inhibits Mo-DC TLR-induced cytokines, co-stimulation of NOD2 results in increased IL-10 and IL-23. IL-12p70 was completely abrogated by 1,25D. 1,25D similarly modulated cytokine production by immune cells in ulcerative colitis patients and healthy controls. Mo-DCs from CD patients heterozygous for NOD2 mutations had a response similar to those from patients without NOD2 mutations. Immune cells from patients homozygous for the $1007 \mathrm{fs}$ mutation were unresponsive to MDP and 1,25D. Our in vitro data support 1,25D as a potential modulator of immunity. However, these results cannot be extrapolated to CD patients without further controlled studies.

\section{INTRODUCTION}

Crohn's disease $(\mathrm{CD})$ is an incurable immune-mediated disorder that can affect any segment of the gastrointestinal tract. The prevailing etiological hypothesis is that a combination of genetic predisposition and environmental factors leads to excessive inflammatory responses toward the intestinal commensal microbiota. ${ }^{1,2}$ Heritability of $\mathrm{CD}$ is relatively high compared with other autoimmune diseases such as ulcerative colitis and rheumatoid arthritis, with a genetic risk ratio estimated at $15-27 .^{3}$ Genome-wide association studies have identified over 160 risk-conferring loci for inflammatory bowel disease (IBD), many of which are shared by $\mathrm{CD}$ and UC. ${ }^{4-6}$ Several of these genes code for molecules involved in host defense against pathogens, such as nucleotide-binding oligomerization domain 2 (NOD2), ATG16L1, and those implicated in the $\mathrm{T}$ helper type 17 (Th17) pathway. ${ }^{1-6}$ Three NOD2 polymorphisms, R702W, G908R, and L1007fs, account for 80\% of $\mathrm{CD}$-associated variants and are established as the most common genetic risk factor for developing CD. ${ }^{7}$
NOD2 recognizes muramyl dipeptide (MDP), a peptidoglycan breakdown product of Gram-positive and Gram-negative bacteria. NOD2 activation promotes receptor interacting protein 2 binding and nuclear factor (NF)- $\mathrm{\kappa B}$ essential modulator ubiquitination, stimulating NF- $\mathrm{KB}$ and mitogenactivated protein kinase activation to induce expression of chemokines and cytokines. The three common genetic variants in the NOD2 gene all result in defective MDP responses. ${ }^{8}$ Innate immune responses mediated by NOD2, including production of pro-inflammatory cytokines and antimicrobial peptides, as well as induction of autophagy, are thought to have a central role in the pathogenesis of $\mathrm{CD}$ and these processes are impaired in patients homozygous for NOD2 mutations. ${ }^{9-15}$

Bearing a NOD2 mutation alone is insufficient for developing CD. A combination of susceptibility alleles that modulate microbial handling and the immune response may lead to CD subsequent to exposure to yet unknown, environmental trigger(s). Environmental factors linked with susceptibility to $\mathrm{CD}$ include smoking and some intestinal infections. Diet, ${ }^{1}$ McGill Center for IBD Research, Research Institute of the McGill University Health Center, McGill University, Montreal, Quebec, Canada and ${ }^{2}$ Department of Physiology,
Faculty of Medicine, McGill University, Montreal, Quebec, Canada. Correspondence: EG Seidman (ernest.seidman@mcgill.ca)

Received 6 March 2013; accepted 6 March 2014; published online 30 April 2014. doi:10.1038/mi.2014.30 
certain drugs, geography, social status, stress, enteric microbiota, and intestinal permeability are also considered potential risk factors. ${ }^{16}$

There is a geographical pattern to the worldwide prevalence of $\mathrm{CD}$. Increasing rates at higher latitudes have been linked to lower cutaneous vitamin D (vD) synthesis in Northern Europe $^{17}$ and North America. ${ }^{17,18}$ There is a strong negative correlation between the frequency of $\mathrm{CD}$ and exposure to sunlight, as represented by the distance from the equator. ${ }^{19}$ Moreover, vD deficiency is more common in both adult and pediatric-onset IBD, especially CD. ${ }^{19,20}$ Higher circulating levels of $\mathrm{vD}$ were recently shown to be protective against the risk for $\mathrm{CD} .{ }^{21}$ Few studies have directly examined the effect of $\mathrm{vD}$ supplementation on $\mathrm{CD}$ activity. ${ }^{22}$ In one recent study, $\mathrm{CD}$ patients receiving 1200 IU daily of vitamin D3 had a lower relapse rate compared with those treated with placebo. ${ }^{23}$

The impact of $\mathrm{vD}$ on gut homeostasis has been corroborated by studies in experimental animals. VDR-deficient mice develop lethal enterocolitis ${ }^{24}$ and CYP27B1 knockout mice are more susceptible to colitis. ${ }^{25}$ Moreover, 1,25D (hormonal form of $\mathrm{vD}$ ) supplementation has been shown to abrogate experimental colitis. ${ }^{26,27}$

$\mathrm{vD}$ regulates adaptive immunity and has inhibitory effects on Th1 $1^{28-30}$ and Th17 pathways. ${ }^{28-32} \mathrm{vD}$ increases microbial cell killing by inducing autophagy and stimulating the production of antimicrobial peptides, ${ }^{28}$ two key processes in which NOD2 also has a role. ${ }^{1,2,11,12}$ The importance of $\mathrm{vD}$ on innate immunity was recently emphasized by our discovery of its capacity to directly induce NOD2 gene expression, the most common susceptibility gene for $\mathrm{CD} .^{12}$ The diverse roles of $\mathrm{vD}$ in immune regulation, along with the epidemiological studies cited above, have led to the hypothesis that $\mathrm{vD}$ deficiency may be a key environmental risk factor for $\mathrm{CD} .{ }^{31} \mathrm{VD}$ deficiency may be associated with suboptimal NOD2 expression that could result in impaired immune response to microbial activators. We therefore investigated the effect of $\mathrm{vD}$ on immune responses induced by Toll-like receptor (TLR) and NOD2 ligation and whether vD-induced NOD2 has the ability to modulate TLR-induced cytokines in peripheral blood mononuclear cells (PBMCs) and monocyte-derived dendritic cells (Mo-DCs) from CD patients. The effect of $\mathrm{vD}$ was also assessed using immune cells from patients with UC and from healthy individuals. The influence of NOD2 mutations on the effects of $\mathrm{vD}$ was also examined. In order to determine the effects of $\mathrm{vD}$ on cytokine gene expression and some of the downstream signaling molecules involved, we employed the THP-1 cell line.

\section{RESULTS}

A total of 113 samples were obtained from 96 patients with an established diagnosis of CD. Details about the characteristics of their disease are shown in Table 1. Over half of the cohort had pediatric-onset disease (A1). The majority had ileocolonic involvement and a mucosal inflammatory disease phenotype. However, about $25 \%$ had a more severe disease phenotype, with either a stricturing or a penetrating/fistulizing behavior. About one-third had significant perianal involvement, with either
Table 1 Description of the IBD patients and controls studied

\begin{tabular}{lccc}
\hline & CD & UC & Controls \\
\hline$N$ & 113 & 20 & 17 \\
Females, $n(\%)$ & $54(47.8)$ & $13(65.0)$ & $10(58.8)$ \\
Age, median (range) & $22(8-63)$ & $22(19-66)$ & $30(21-58)$
\end{tabular}

Age at diagnosis

$\begin{array}{lcrr}\text { A1 }(<16) & 70(61.9) & 5(25.0) & - \\ \text { A2 (17-40) } & 36(31.9) & 13(65.0) & - \\ \text { A3 }(>40) & 7(6.2) & 2(10.0) & -\end{array}$

$\begin{array}{llrr}\text { Treatment } & & & \\ \text { None } & 17(15.0) & 2(10.0) & 17(100) \\ \text { 5-ASA alone } & 10(8.8) & 4(20.0) & - \\ \text { Thiopurine or methotrexate } & 55(48.7) & 11(55.0) & - \\ \text { TNF inhibitor } & 34(30.0) & 7(35.0) & - \\ \text { Oral corticosteroids } & 11(9.7) & 1(5.0) & -\end{array}$

Disease location: $C D$

L1-terminal ileum

L2-colon

L3-ileocolonic

L4-upper Gl tract

$\begin{array}{ccc}5(4.4) & - & - \\ 35(31.0) & - & - \\ 73(64.6) & - & - \\ 33(29.2) & - & -\end{array}$

$\begin{array}{llrl}\text { Disease location: UC } & & & \\ \text { E1-proctitis } & - & 1(5.0) & - \\ \text { E2-left-sided colitis } & - & 5(25.0) & - \\ \text { E3-pancolitis } & - & 10(50.0) & - \\ \text { E4-proximal colitis } & - & 4(20.0) & -\end{array}$

Disease behavior: $C D$

\begin{tabular}{lccc} 
B1 - Inflammatory & $84(74.3)$ & - & - \\
B2 - Stricturing & $14(12.4)$ & - & - \\
B3 - Penetrating & $15(13.3)$ & - & - \\
Perianal disease & $37(32.7)$ & - & - \\
Vitamin D supplementation (\%) & $40(35.3)$ & $6(30)$ & $2(12)$ \\
Vitamin D status, $n$ & 85 & 11 & - \\
Sufficient, $n$ (\%) & $37(43.5)$ & $3(27.3)$ & - \\
Insufficient, $n(\%)$ & $34(40.0)$ & $4(36.4)$ & - \\
Deficient, $n(\%)$ & $14(16.5)$ & $4(36.4)$ & - \\
\hline
\end{tabular}

$\mathrm{CD}$, Crohn's disease; IBD, inflammatory bowel disease; UC, ulcerative colitis.

abscess or fistula formation. Circulating $25(\mathrm{OH}) \mathrm{D}$ levels were sufficient $\left(>75 \mathrm{nmoll}^{-1}\right)$ in less than half $(43.5 \%)$. Thus, despite the fact that $35 \%$ of patients were supplemented with $\mathrm{vD}$ at the time of blood draw, the majority of the cohort had vD insufficiency or deficiency. Among those not taking supplementation, the proportion of patients having inadequate 
levels of 25(OH)D was $63.7 \%$. Among the UC patients recruited, the prevalence of vD insufficiency and deficiency was similar (Table 1).

\section{Pattern recognition receptor (PRR)-induced cytokine secretion in PBMCs from CD patients and controls}

We first examined the array of CD PBMC cytokine induction in response to PRR stimulation (Figure 1a). Unstimulated cells showed very low baseline cytokine secretion. IL-12/IL-23p40 and IL-10 were induced by stimulation of each PRR ligand alone. TNF- $\alpha$ release was increased by all TLR ligands, but not by NOD2 stimulation. Only TLR4 (lipopolysaccharide (LPS)) and TLR7/8 (R848) stimulation resulted in increased IL-23
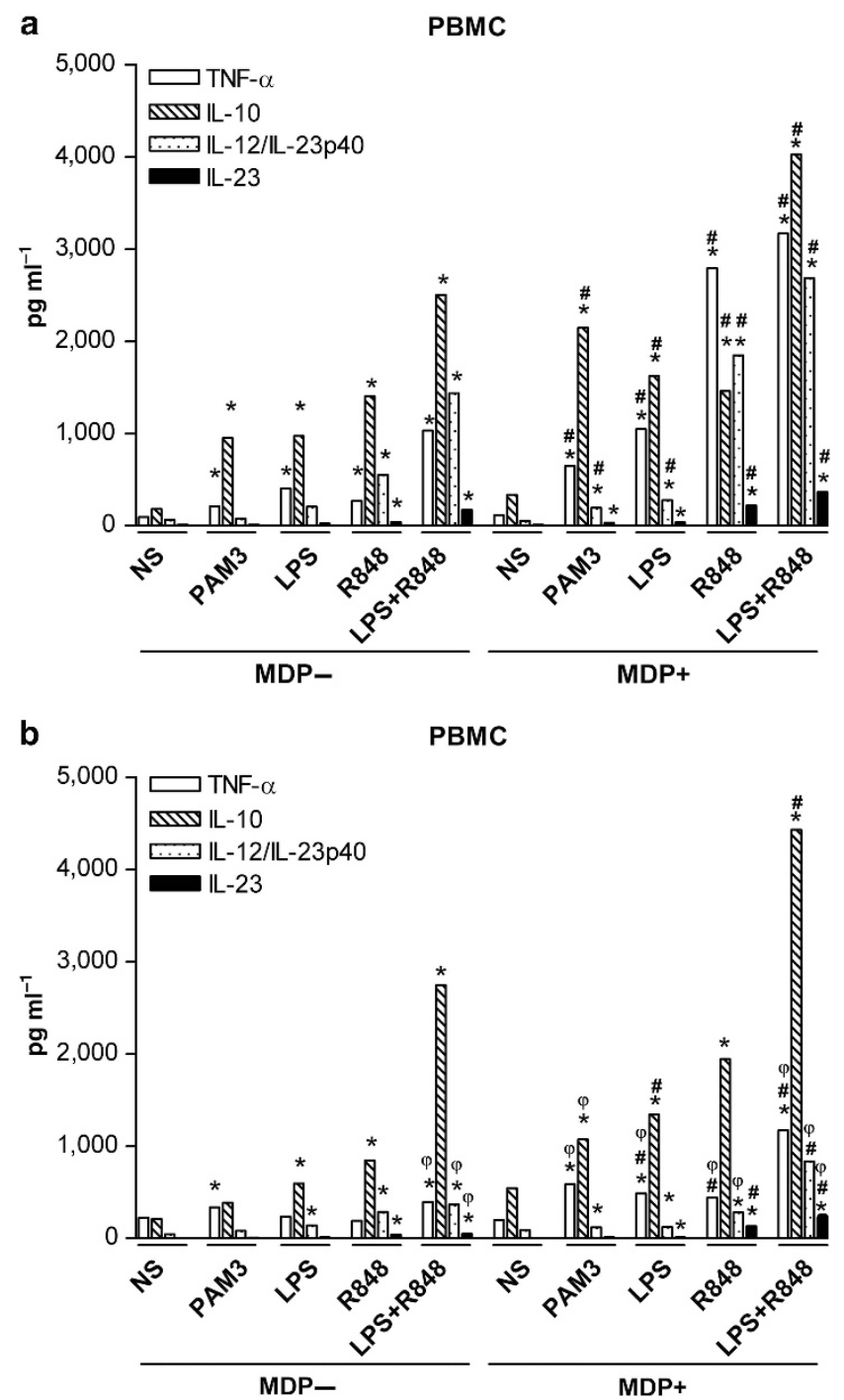

Figure 1 Pattern-recognition receptor-induced cytokine production in peripheral blood mononuclear cells (PBMCs) from Crohn's disease (CD) patients (a) and controls (b). PBMCs were left untreated non-stimulated (NS) or stimulated with the indicated ligands for $24 \mathrm{~h}$. ${ }^{*} P<0.05$ compared with paired NS samples, ${ }^{\#} P<0.05$ compared with paired stimulated samples in the absence of muramyl dipeptide (MDP), and ${ }^{\oplus} P<0.05$ compared with values from $C D$ patients stimulated using the same ligands. Data are the median of 7-25 samples for $C D$ and 3-15 for controls. secretion. Flagellin, initially employed to stimulate TLR5, only resulted in minimal levels of the cytokines analyzed (data not shown). In order to simulate in vivo microbial triggering of multiple PRR, cells were stimulated with two TLR ligands (LPS + R848) or by the combination of NOD2 and TLR activation (MDP + LPS and MDP + LPS + R848). All cytokines were increased by simultaneous stimulation with two ligands compared with single receptor triggering (Figure 1a). High levels of IL-12/IL-23p40 and IL-23 were measured when TLR4 and TLR7/8 were co-stimulated. Stimulation with NOD2 and TLR ligands increased the levels of all cytokines, particularly TNF- $\alpha$ and IL-10. The pattern of cytokine production by control PBMCs was similar to that observed using CD PBMCs (Figure 1b). However, control PBMCs secreted lower amounts of TNF- $\alpha$, IL-12/IL-23p40, and IL-23 compared with those from $\mathrm{CD}$ patients when stimulated with two or more ligands.

\section{1,25D decreased TLR-induced cytokine production by PBMCs from CD patients.}

1,25D pretreatment decreased the production of TLR2-, TLR4-, and TLR7/8-induced cytokines (Figure 2). IL-12/IL-23p40 levels were reduced by $45-56 \%$, whereas those of IL-23 were reduced by $64-70 \%$. IL-10 was less affected, being decreased by up to $25 \%$. Interestingly, $1,25 \mathrm{D}$ did not reduce TLR2-induced IL-10 levels. TNF- $\alpha$ levels were only marginally reduced upon TLR7/8 triggering and slightly decreased by about $25 \%$ by $1,25 \mathrm{D}$ in cells activated with LPS or PAM3. Cytokine production was more markedly decreased by $1,25 \mathrm{D}$ in PBMCs upon simultaneous stimulation with TLR4 and TLR7/8.

\section{1,25D differentially modulated NOD2- and TLR-induced cytokine production of PBMCs from CD patients}

The effect of 1,25D on NOD2-induced cytokine secretion by PBMCs was quite divergent from that of TLR. MDP-induced production of IL-23, IL-10, and TNF- $\alpha$ was increased by $1,25 \mathrm{D}$ pretreatment, whereas that of IL-12/IL-23p40 was not changed (Figure 3). MDP-induced cytokine levels remained low, consistent with the fact that MDP alone is a weak activator of cytokine production. NOD2 activation can strongly amplify the responses provoked by TLR. ${ }^{9}$ We therefore examined the effects of 1,25D in the presence of MDP. PBMCs stimulated with NOD2 in addition to TLR4 behaved more like cells stimulated with the former. 1,25D caused a comparable enhancement of TNF- $\alpha$ upon NOD2 + TLR4 triggering. IL-23 production was slightly increased. On the other hand, IL-12/IL-23p40 production was inhibited, whereas IL-10 induction was not affected by 1,25D. Addition of NOD2 stimulation to combined TLR4 and TLR7/8 triggering led to a secretion pattern more similar to TLR4 + TLR7/8, but the magnitude of inhibition was slightly attenuated overall. 1,25D did not affect TNF- $\alpha$ levels, whereas it decreased the other cytokines by $26-51 \%$. In summary, $1,25 \mathrm{D}$ did not change the synergy between MDP and LPS for IL-10 and IL-23, and it enhanced TNF- $\alpha$ while decreasing IL-12/IL-23p40. In the presence of multiple ligands, $1,25 \mathrm{D}$ was mainly inhibitory. 


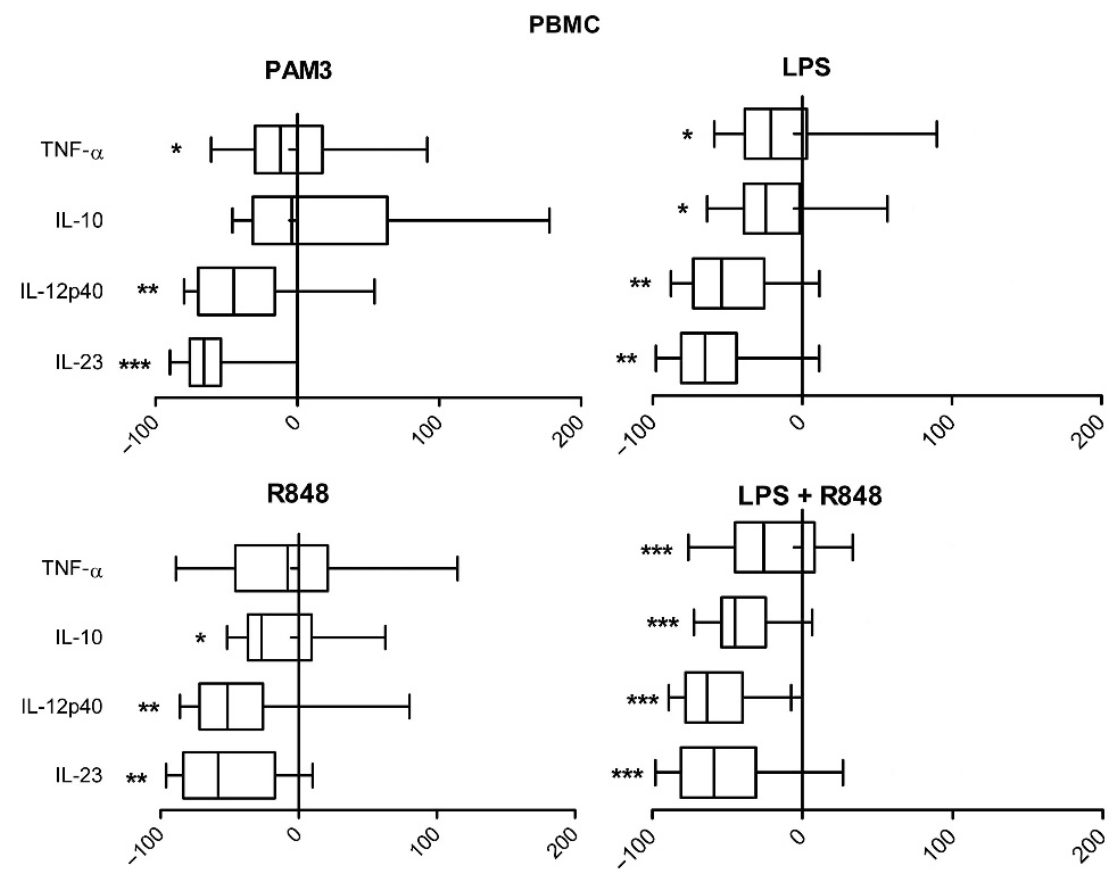

Figure 2 1,25D decreased Toll-like receptor (TLR)-induced cytokine production by peripheral blood mononuclear cells (PBMCs) in Crohn's disease (CD) patients. PBMCs from patients with CD were pretreated with vehicle or 1,25D for $20 \mathrm{~h}$. PBMCs were then stimulated with TLR ligands for $24 \mathrm{~h}$. Box and whisker plots: vertical bars are medians and edges of the box are the 75th and 25th percentiles, lines extending from the box represent the 10th and 90 th percentiles. Data represent the percentage change in cytokine production induced by 1,25D for 12-24 samples for PAM3 and R848, 26-40 samples for lipopolysaccharide (LPS), and 45-48 for LPS + R848. Differences in cytokine levels were analyzed using the Mann-Whitney test. ${ }^{*} P<0.05$, ${ }^{* \star} P<0.01$, and ${ }^{* * *} P<0.001$ compared with paired untreated samples.

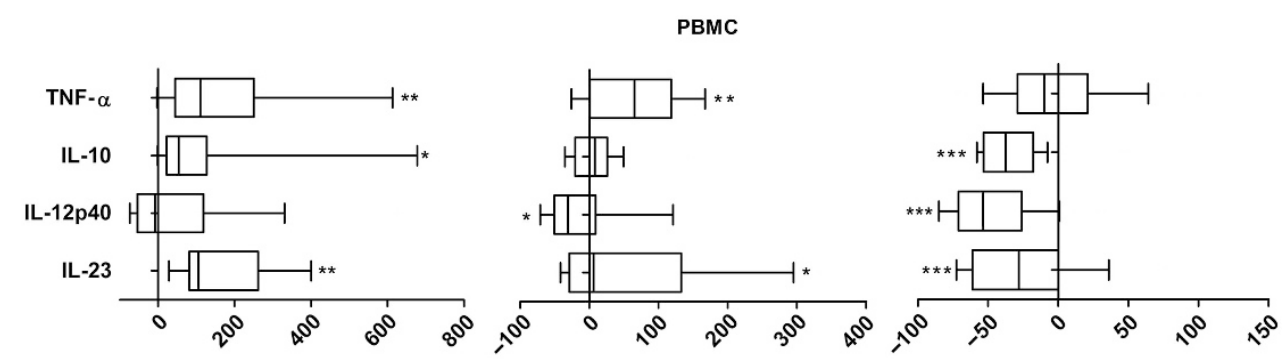

Figure 3 1,25D differentially modulated nucleotide-binding oligomerization domain 2 (NOD2) and Toll-like receptor (TLR) induced cytokine production in Crohn's disease (CD). Peripheral blood mononuclear cells (PBMCs) from patients with CD were pretreated with vehicle or 1,25D for 20 h. PBMCs were then stimulated with NOD2 and TLR ligands for $24 \mathrm{~h}$. Data represent the percentage change in cytokine production induced by 1,25D for 12-23 samples for muramyl dipeptide (MDP), 27-33 samples for MDP + LPS (lipopolysaccharide), and 39-50 samples for MDP + LPS + R848. Differences in cytokine levels were analyzed using the Mann-Whitney test. ${ }^{\star} P<0.05$, ${ }^{\star \star} P<0.01$, and ${ }^{* \star *} P<0.001$ compared with paired untreated samples.

\section{Effect of 1,25D on TLR- and NOD2-induced cytokine production by Mo-DCs from CD patients}

We next studied the effect of $1,25 \mathrm{D}$ on Mo-DCs. Single PRR triggering resulted in very low cytokine levels, precluding the investigation of the effect of $1,25 \mathrm{D}$. Simultaneous activation of TLR4 and TLR7/8 in Mo-DCs resulted in the release of high amounts of cytokines. In addition, IL-12p70 production was significantly induced (Figure 4a). 1,25D reduced levels of IL-12/IL-23p40, TNF- $\alpha$, and IL-23 induced by LPS and R848, by $63-67 \%$. As seen with PBMCs, IL-10 production by Mo-DCs was less affected by $1,25 \mathrm{D}(-26 \%)$, whereas IL-12p70 production was completely abrogated by $1,25 \mathrm{D}$ pretreatment (Figure $\mathbf{4 b}$ ).
1,25D led to increased production of TNF- $\alpha$ (Figure 4c), IL-10, and IL-23 (Figure 4d) when MDP was also present. The inhibitory effect of $1,25 \mathrm{D}$ on IL-12p70 was not changed by $1,25 \mathrm{D}$ when NOD2 was also triggered. These data show that, in response to multiple co-stimulations including NOD2, 1,25D led to increased production of IL-10, IL-23, and TNF- $\alpha$.

\section{Influence of NOD2 genotype on 1,25D effect on TLR- and} NOD2-induced cytokine production

The NOD2 genotype was available for $65 \mathrm{CD}$ patients. NOD2 mutations were identified in 23 patients (35.4\%): 7 for SNP-8 (R702W), 8 for SNP-12 (G908R), and 8 for SNP-13 (1007 fs). 
a
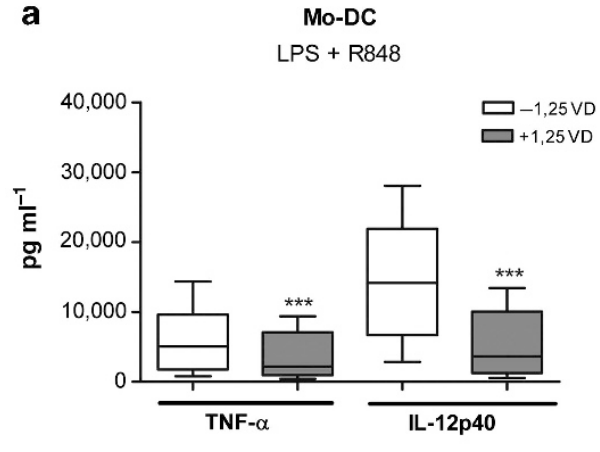

c

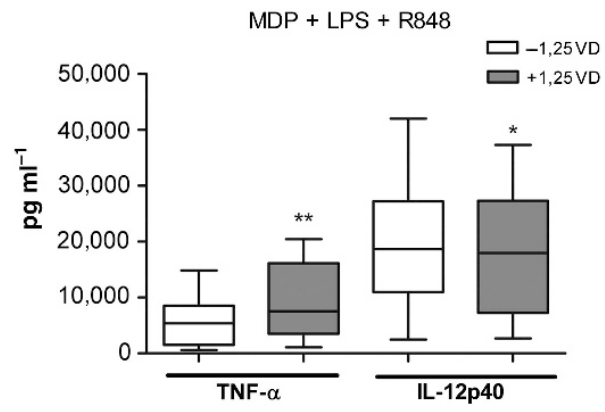

b

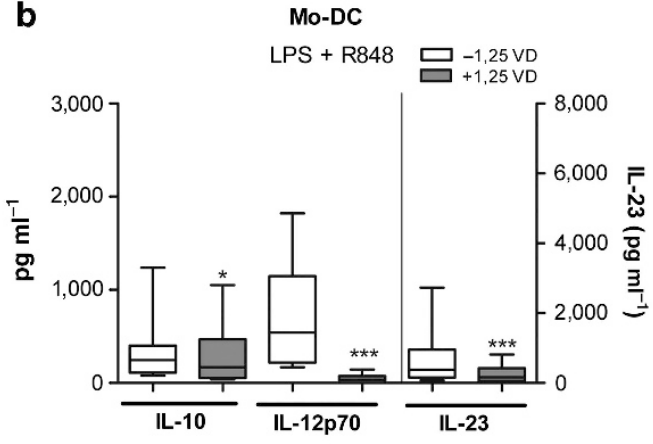

d
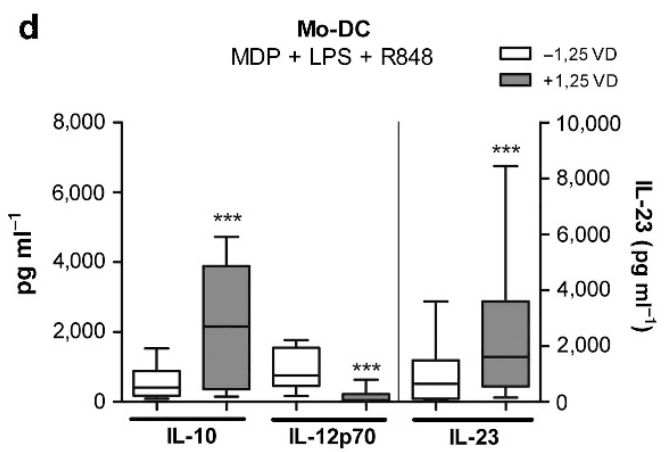

Figure 4 Effect of 1,25D on Toll-like receptor (TLR) and nucleotide-binding oligomerization domain 2 (NOD2) induced cytokine production by monocyte-derived dendritic cells (Mo-DCs) in Crohn's disease (CD) patients. Effect of 1,25D on TLR-induced tumor necrosis factor-alpha (TNF- $\alpha$ ) and interleukin (IL)-12/IL-23p40 (a) and IL-10, IL-12p70, and IL-23 (b) production by Mo-DCs from CD patients. Effect of 1,25D on TNF- $\alpha$ and IL-12/IL-23p40 (c) and IL-10, IL-12p70, and IL-23 (d) production in the presence of TLR and NOD2 activation. Data represent cytokine levels from 26-32 paired samples pretreated with vehicle or $1,25 \mathrm{D}$. ${ }^{\star} P<0.05,{ }^{* *} P<0.01$, and ${ }^{* * *} P<0.001$ compared with paired untreated samples.

One each was homozygous for R702W and 1007 fs. Another patient carried a double mutation (R702W/1007 fs). In comparison, NOD2 mutations were found in only one UC patient (out of 10) and in none of the healthy individuals (out of nine). The effect of NOD2 mutations on 1,25D modulation of cytokine release was analyzed using cells from $\mathrm{CD}$ patients. As expected, 1,25D exerted very comparable inhibitory effects on cytokine production by PBMCs induced by TLR ligands in patients having NOD2 wt and those carrying one NOD2 mutated allele (data not shown). In response to NOD2dependent signaling, PBMCs from patients heterozygous for NOD2 had blunted TNF- $\alpha$ and IL-10 responses when stimulated with MDP + LPS in the presence of 1,25D, although the difference did not reach significance (Figure 5a). IL-23 production by PBMCs was induced in only half of the patients heterozygous for NOD2, whereas IL-12p40 was decreased in those patients (Figure 5a). We then analyzed the effect of the different mutations on response. Patients heterozygous for the 1007 fs mutation had a decreased response compared with those bearing the G908R or the R702W mutations (Supplementary Figure $\mathbf{S} 1$ online). When PBMCs were stimulated by MDP + LPS + R848, 1,25D similarly decreased IL-10, IL-23, and IL-12p40 in patients heterozygous for NOD2 and in those bearing NOD2 wt (Figure 5b). Cells from R702W/1007 fs and R702W/R702W patients had a response similar to those heterozygous for NOD2. The situation was quite different for the patient homozygous for the $1007 \mathrm{fs}$ mutation, whose cells completely lacked any response to MDP.
The effect of 1,25D was also comparable for Mo-DCs from patients with NOD2 wt and those heterozygous for NOD2. As Mo-DCs could be generated only when the cell number was sufficient, fewer samples were available than for PBMCs. The effect of carrying different mutated alleles on Mo-DCs was essentially the same as for PBMCs, as only cells from the patient harboring the $1007 \mathrm{fs} / 1007$ fs genotype displayed a different response to 1,25D in the presence of MDP (Figure 5c). In this case, the effect of $1,25 \mathrm{D}$ in the presence of MDP was very similar to the effect observed with TLR agonists alone. In summary, Mo-DCs from patients heterozygous for NOD2 responded to $1,25 \mathrm{D}$ similarly to those without mutations.

\section{Effect of 1,25D on TLR- and NOD2-induced cytokine production in patients with $\mathrm{CD}, \mathrm{UC}$, and in controls}

We examined whether 1,25D exerted similar immunomodulatory effects in cells from patients with UC and in those from healthy individuals. The effect of $1,25 \mathrm{D}$ on cytokine production induced by MDP + LPS was comparable between CD patients bearing NOD2 wt, UC patients, and control individuals (Figure 6a). When PBMCs from $\mathrm{CD}$ patients without NOD2 polymorphisms and from UC patients were stimulated with MDP + LPS + R848, 1,25D also exerted similar effects on cytokine production (Figure $6 \mathbf{b}$ ). The effect of $1,25 \mathrm{D}$ on PBMCs from control individuals was more variable. Globally, 1,25D was less inhibitory in control cells. IL-23 and IL-12p40 production was not affected in controls, whereas it was decreased in most patients with CD and UC. TNF- $\alpha$ 
PBMC

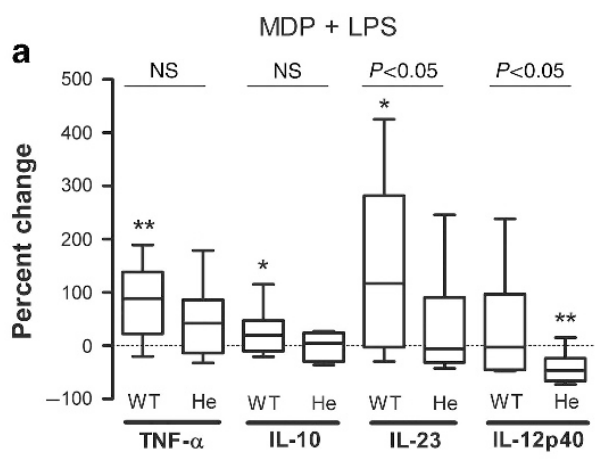

PBMC

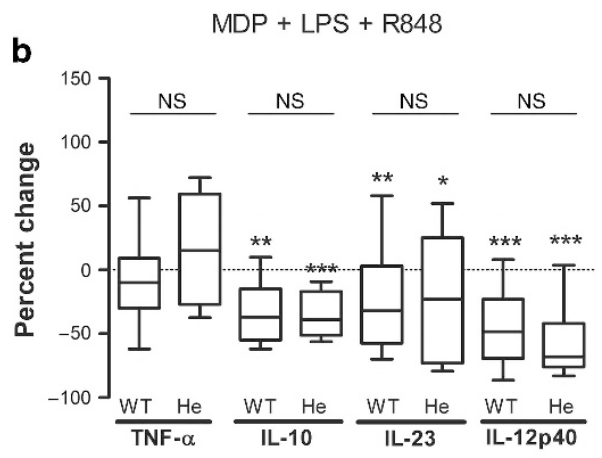

b

C

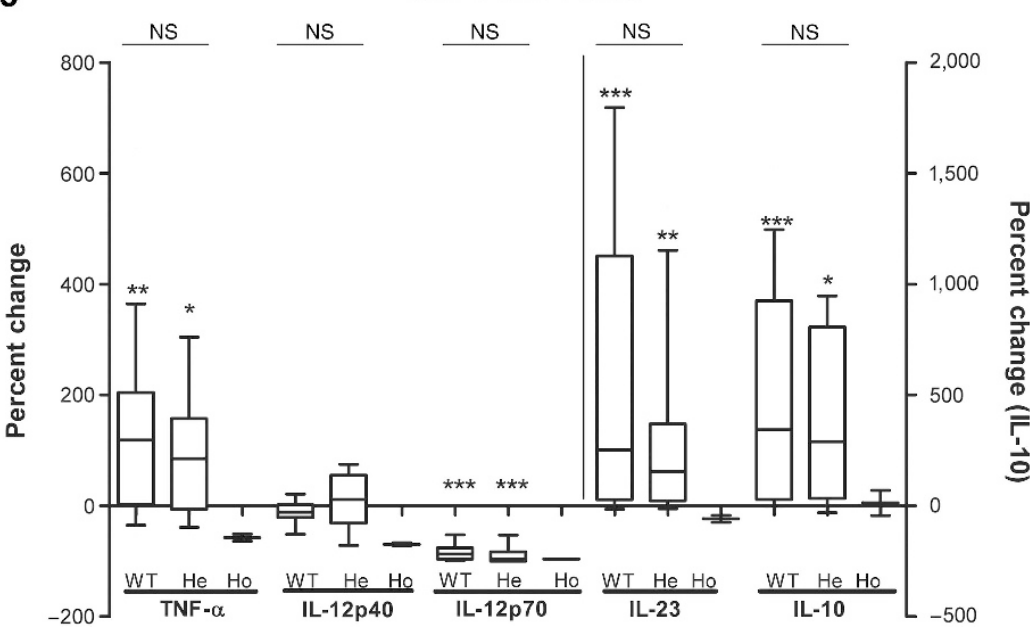

Figure 5 Influence of nucleotide-binding oligomerization domain 2 (NOD2) genotype on the effect of 1,25D on cytokine production in Crohn's disease. (a) Influence of NOD2 genotype on the effect of 1,25D on cytokine production induced by co-activation of NOD2 and Toll-like receptor 4 (TLR4) in peripheral blood mononuclear cells (PBMCs) ( $n=12-14$ NOD2 wt and 12-15 heterozygous patients). (b) Influence of NOD2 genotype on the effect of $1,25 \mathrm{D}$ on cytokine production induced by co-activation of NOD2, TLR4, and TLR7/8 in PBMCs ( $n=26-32$ NOD2 wt and 13-15 heterozygous patients). (c) Influence of NOD2 genotype on the effect of 1,25D on cytokine production by monocyte-derived dendritic cells (Mo-DCs). ( $n=15-18$ NOD2 wt, 10-12 heterozygous, and 2 homozygous patients) WT: NOD2 wild type, He: heterozygous NOD2, Ho: homozygous for 1007 fs mutation. ${ }^{*} P<0.05$, ${ }^{* \star} P<0.01$, and ${ }^{* * *} P<0.001$ compared with paired untreated samples.

production was unchanged in the three groups, but there was an increased response in controls compared with CD patients.

In Mo-DCs, there was no difference between groups on the basis of analysis of variance, although cells from control individuals tended to display a higher percentage increase in production following $1,25 \mathrm{D}$ treatment. This apparent higher response is not caused by higher levels of cytokine in controls, as cytokine levels in cells stimulated after 1,25D pretreatment were not higher in the media from Mo-DCs from control individuals (Figures $7 \mathbf{a}$ and $\mathbf{b}$ ). However, Mo-DCs from controls produced lower cytokine levels when stimulated with MDP + LPS + R848 in the absence of $1,25 \mathrm{D}$. This was particularly the case for TNF- $\alpha$ (Figure 7a) and IL-23 (Figure $7 \mathbf{b}$ ).

\section{Effect of 1,25D on cytokine gene expression and NF-kB downstream signaling}

The mechanisms involved in $\mathrm{vD}$-induced alterations in cytokine production were first investigated by examining
IкB- $\alpha$ using patient PBMCs and Mo-DCs by western blot (Figure 8). 1,25D increased basal IкB- $\alpha$ levels. However, upon stimulation with MDP + LPS + R848, IкB- $\alpha$ degradation was induced irrespective of VD exposure. In addition, 1,25D increased p-ERK phosphorylation levels in PBMCs but not in Mo-DCs.

Further investigation of the mechanisms involved in cytokine modulation by $1,25 \mathrm{D}$ vD was not feasible because of the limited number of cells available in a finite group of IBD patients. Therefore, in order to examine the signaling pathways involved, we determined the quantitative gene expression profiles of undifferentiated THP-1 cells under similar activation protocols. We found that gene expression of NOD2 and TNF- $\alpha$ was enhanced by $\mathrm{VD}$ in the presence of MDP alone, or in combination with TLR ligands (Supplementary Figure S2). In terms of downstream effector molecules, we also employed the THP-1 cell line to examine NF- $\kappa B$ signaling. As shown in Supplementary Figure S3, 1,25D with TLR activation enhanced NF- $\kappa \mathrm{B}$ gene expression. 
a

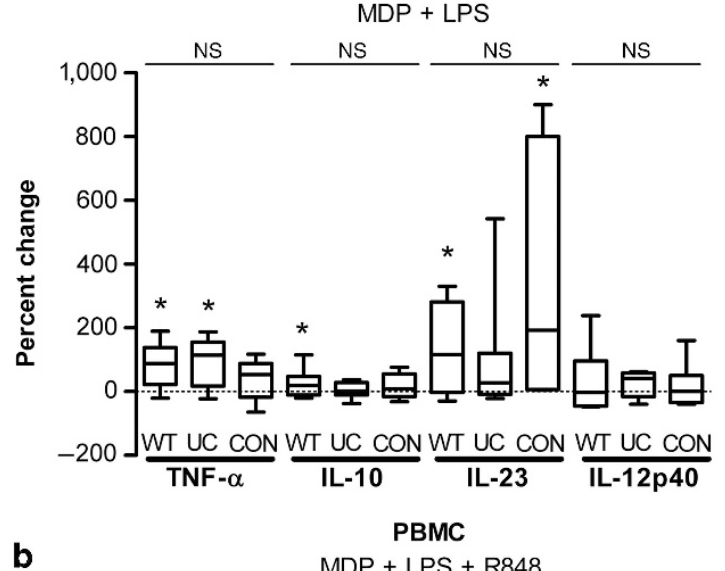

b $\quad$ MDP + LPS + R848

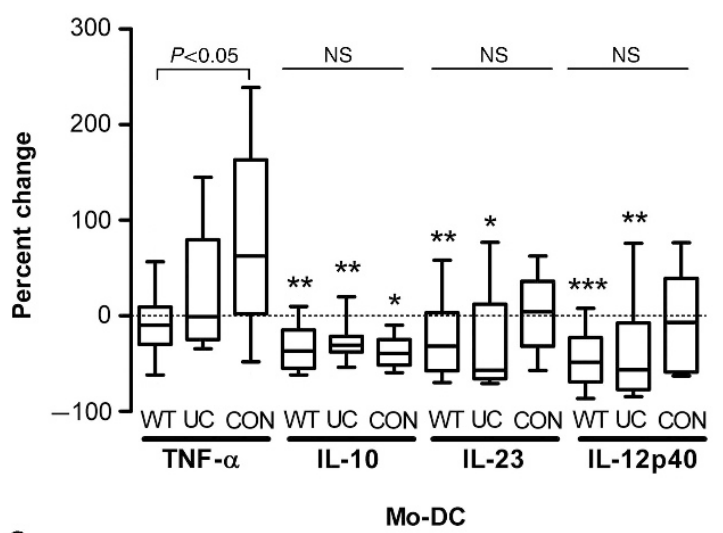

c

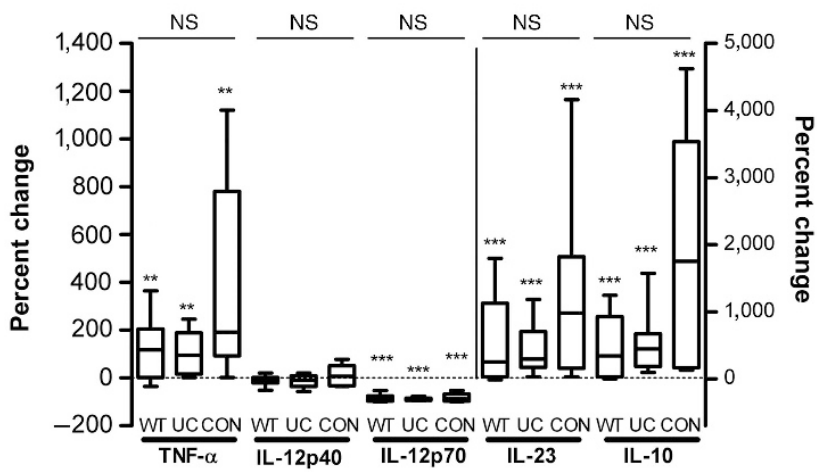

Figure 6 Effect of 1,25D on Toll-like receptor (TLR) and nucleotidebinding oligomerization domain 2 (NOD2) induced cytokine production in Crohn's disease patients bearing wild-type NOD2 (CDwt), ulcerative colitis (UC), and controls. (a) Effect of 1,25D on cytokine production induced by co-activation of NOD2 and TLR4 in peripheral blood mononuclear cells (PBMCs) from CDwt, UC, and controls ( $n=7 \mathrm{UC}$ and 7-9 controls). (b) Effect of 1,25D on cytokine production induced by co-activation of NOD2, TLR4, and TLR7/8 in PBMCs from CDwt, UC, and controls. ( $n=9-12$ UC and 10-13 controls). (c) Effect of 1,25D on cytokine production induced by co-activation of NOD2, TLR4, and TLR7/8 in monocyte-derived dendritic cells (Mo-DCs) from CDwt, UC, and controls ( $n=11-12$ UC and 11-12 controls). ${ }^{*} P<0.05,{ }^{* *} P<0.01$, and ${ }^{* \star *} P<0.001$ compared with paired untreated samples.

\section{Correlation between the effect of 1,25D on cytokine production and circulating 25(OH)D}

Correlation analysis was performed for all cytokines following stimulation with LPS + R848, MDP + LPS, and MDP + LPS +
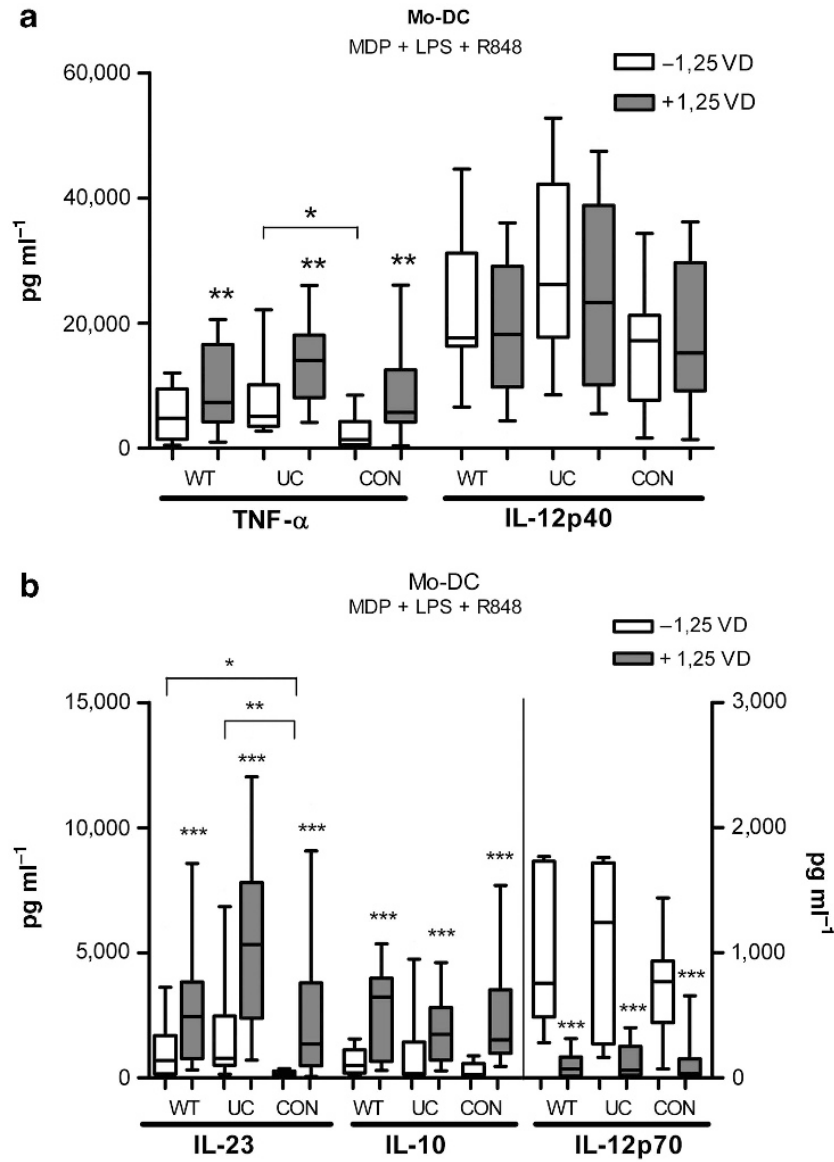

Figure 7 Cytokine levels in supernatants of paired monocytederived dendritic cells (Mo-DCs) stimulated with nucleotide-binding oligomerization domain 2 (NOD2), Toll-like receptor (TLR)4, and TLR7/8 ligands following pretreatment with vehicle or $1,25 \mathrm{D}$. (a) TNF- $\alpha$ and IL-12p40. (b) IL-23, IL-10, and IL-12p70. ${ }^{*} P<0.05$, ${ }^{* *} P<0.01$, and ${ }^{* \star *} P<0.001$ compared with paired untreated samples.

R848 in PBMCs and with MDP + LPS + R848 in Mo-DCs. The only data approaching statistical significance were for LPS + R848- and MDP + LPS + R848-induced TNF- $\alpha$ production ( $r=0.26, P=0.117$, and $r=0.36, P=0.061$, respectively) (Supplementary Figure S4).

\section{DISCUSSION}

$\mathrm{CD}$ is characterized by chronic inflammation, primarily involving Th1 and Th17 cytokines. ${ }^{1,2}$ There is growing evidence that impaired innate immune response has a key role in the pathogenesis of CD. ${ }^{13-15,32,33}$ Loss of tolerance to gut microorganisms is believed to have a role in the pathogenesis of CD, and genome-wide association studies have identified several susceptibility genes implicated in defective microbial recognition., ${ }^{1,5,6}$

Numerous studies confirm that $1,25 \mathrm{D}$ enhances the innate immune response, whereas it exerts an inhibitory action on the adaptive immune system. ${ }^{28,29}$ Notably, 1,25D was reported to have inhibitory effect on $\mathrm{Th}^{29,30}$ and Th17 pathways, ${ }^{29,34,35}$ while promoting Th2 and Treg phenotypes. ${ }^{29}$ The antimicrobial actions of $1,25 \mathrm{D}$ are mediated via induction of the antimicrobial peptides $\beta$-defensin 2 and cathelicidin and that of 

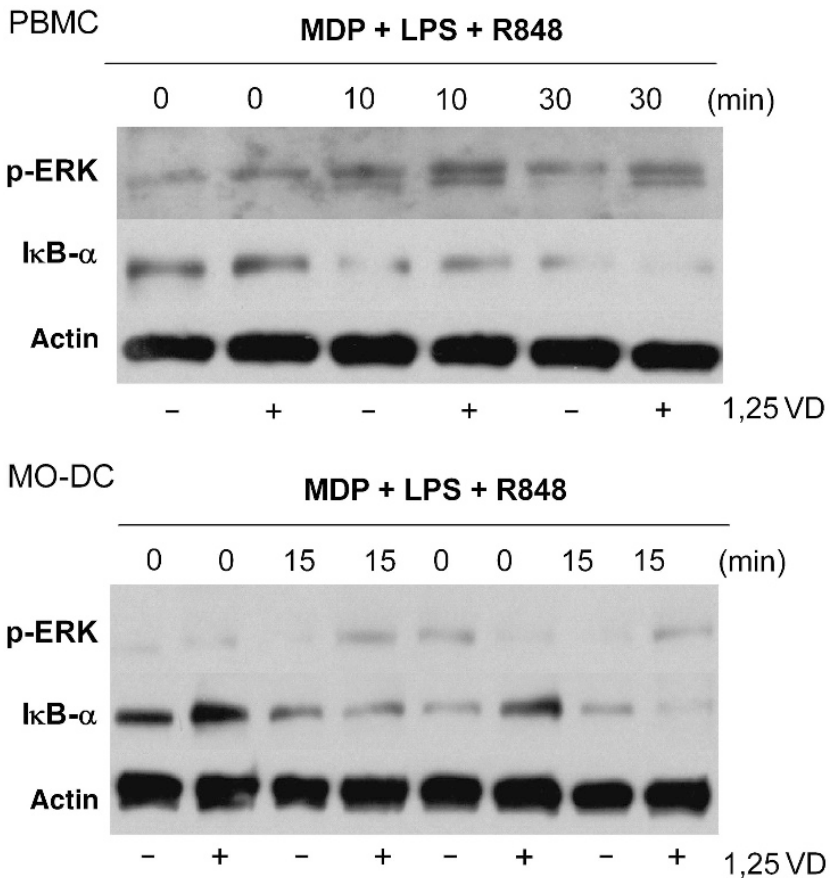

Figure 8 Effect of $1,25 \mathrm{D}$ on $\mathrm{I} \kappa \mathrm{B}-\alpha$ and $\mathrm{p}$-ERK expression in peripheral blood mononuclear cells (PBMCs) and monocyte-derived dendritic cells (Mo-DCs) in Crohn's disease patients. PBMCs and Mo-DCs were treated with 40 and $15 \mathrm{~nm} 1,25 \mathrm{D}$, respectively, for $20 \mathrm{~h}$ followed by treatment with muramyl dipeptide (MDP) $\left(5 \mu \mathrm{g} \mathrm{ml}^{-1}\right)$, lipopolysaccharide (LPS) $\left(100 \mathrm{ng} \mathrm{ml}^{-1}\right)$, and $\mathrm{R} 848\left(2 \mu \mathrm{g} \mathrm{ml}^{-1}\right)$ for the indicated times. Cells were lysed and western blot analyses were performed, using anti-IкB- $\alpha, p-E R K$, and $\beta$-actin antibodies. Representative western blots from 3 PBMC and 2 Mo-DC experiments are shown.

PRR NOD2, ${ }^{12,28}$ key factors implicated in the induction of autophagy. $^{13-15,36}$

Epidemiological data implicate low vD levels with several autoimmune and chronic inflammatory disorders. ${ }^{37,38}$ Several lines of evidence support vD deficiency as an environmental risk factor for developing $\mathrm{CD},{ }^{31}$ including the North-South gradient of incidence and prevalence ${ }^{17,18}$ and the presence of low serum concentrations of $\mathrm{vD}$ in many IBD patients. As well, there is an inverse correlation between elevated vD concentrations and risk for $\mathrm{CD} .^{21}$ Moreover, vD supplementation was observed to reduce disease activity in patients with $\mathrm{CD},{ }^{23}$ as well as ameliorate experimental models of IBD. ${ }^{26,27}$ In this study, we did not find any correlation between the effect of $1,25 \mathrm{D}$ on cytokine production and the patients' circulating 25(OH)D level. However, the patients' PBMCs were tested in vitro, removed from the patients' circulating milieu. Other factors may explain this observation. vD-sufficient and -deficient patients could have different cytokine responses at lower doses of $1,25 \mathrm{D}$, but this was not specifically assessed in this study. It is also possible that more patients are necessary to assess the effect of patients' $25(\mathrm{OH}) \mathrm{D}$ levels on the regulation of cytokine production by $1,25 \mathrm{D}$.

In this study, we examined the effect of $1,25 \mathrm{D}$ on TLR- and NOD2-induced cytokine responses in CD patients. $1,25 \mathrm{D}$ decreased TLR-induced cytokine secretion in CD, in line with previous studies in the unaffected general population. ${ }^{39,40}$ This is also in accordance with decreased LPS-induced TNF production by a vD analog in PBMCs from patients with CD. ${ }^{41}$

The functional data presented here complement our recent discovery of NOD2 gene induction by $1,25 \mathrm{D} .{ }^{12}$ In agreement with the expectation that NOD2 function is increased by $\mathrm{vD}$, we showed that pretreatment with $1,25 \mathrm{D}$ resulted in enhanced MDP-induced TNF- $\alpha$, IL-10, and IL-23 production. On the other hand, IL-12/IL-23p40 release was decreased by $1,25 \mathrm{D}$. Confrontation in vivo with bacteria will most likely result in simultaneous triggering of multiple PRR, potentially for sustained periods of time. Therefore, our in vitro data cannot be extrapolated to the situation in vivo. Cooperation between PRRs is required to mount an efficient innate response, to afford protection against pathogens. ${ }^{42}$ Cells were thus stimulated with MDP in association with one and two TLR ligands. The effect of $1,25 \mathrm{D}$ in this context was more complex. $1,25 \mathrm{D}$ enhanced cytokine production in Mo-DCs and in PBMCs stimulated with NOD2 and TLR4 ligands, whereas it was inhibitory toward PBMCs stimulated with MDP + LPS + $\mathrm{R} 848$. These effects were not specific to $\mathrm{CD}$, as similar responses were observed in patients with UC and in healthy individuals.

How NOD2 mutations predispose to CD is still uncertain. A decreased amount of MDP-induced cytokines in PBMCs and monocytes from patients with NOD2 mutations was documented in several studies.9,43-45 Synergy between TLR and NOD2 is also defective in patients carrying mutated NOD2 alleles. ${ }^{9,44,46}$ Although most functional studies aiming at unraveling the role of NOD2 in CD pathogenesis focus on homozygous carrier of the NOD2 $1007 \mathrm{fs}$ frameshift mutation, this genotype in rarely encountered, representing $<4 \%$ of CD patients. ${ }^{47}$ Studies using cells from CD patients with heterozygous NOD2 genotype generally observed a response similar to that of wild-type patients. ${ }^{43,44}$ Specific differences were sometimes observed, such as decreased MDP-induced IL-10 and increased TNF release by monocytes. ${ }^{45}$ Individuals homozygous for R702W and G908R and compound heterozygous have an intermediate response to MDP. ${ }^{9,43}$ We observed a loss of NOD2 function with low concentrations of MDP, whereas at higher concentrations MDP induced a response in compound heterozygotes. However, no response was seen in a homozygous carrier of the NOD2 $1007 \mathrm{fs}$ mutation, which may be indicative of a decreased sensitivity to MDP rather than a loss of function. ${ }^{9,43}$ Additional studies using adherent-invasive Escherichia coli and Salmonella typhimurium in monocytes and Mo-DCs from CD also pointed toward NOD2 loss of function in individuals carrying the $1007 \mathrm{fs} / 1007$ fs genotype. ${ }^{48,49}$

Our results are in line with previous reports showing that patients homozygous for the $1007 \mathrm{fs}$ mutation are unresponsive to MDP and 1,25D. Response of PBMCs and Mo-DCs to 1,25D and MDP in patients heterozygous for NOD2 mutations was similar to that of cells from wild-type patients. The leading hypothesis concerning $\mathrm{CD}$ pathogenesis implicates defective innate immune function in bacterial processing and clearance, 
resulting in bacterial persistence and mucosal inflammation. Mutations in NOD2 confer an increased susceptibility to CD. Our data raise the possibility that patients who are not homozygous for $1007 \mathrm{fs}$ might benefit from 1,25D as well as those having a deficient innate response without carrying NOD2 mutations. ${ }^{33}$ However, well-designed clinical trials would be needed to properly evaluate this possibility.

The extensive cross-talk between NOD2 and TLR has an important role both in host defense against microbial infection and in the development of autoimmunity. In the present study, we examined the effect of $1,25 \mathrm{D}$ on this cross-talk, under different TLR activation protocols. In addition, we compared response in PBMCs and Mo-DCs from CD patients. The effect of 1,25D on TLR-induced cytokines was clearly inhibitory, particularly toward the pro-inflammatory cytokines IL-12/IL23p40 and IL-23. 1,25D enhanced MDP-cytokine production, except IL-12/IL-23p40, which was inhibited. The effect on the interaction between NOD2 and TLR is complex and dependent on the context of activation and the cell type studied. On coactivation, the effect of $1,25 \mathrm{D}$ was dependent on the activated TLR. Response to MDP + LPS was considerably increased by 1,25D. In contrast, decreased responses were observed upon stimulation via MDP + LPS + R848 in PBMCs. The effect is not solely related to the presence of R848, as PBMCs stimulated with MDP + PAM3 and MDP + R848 also showed this enhancing effect of $1,25 \mathrm{D}$ on NOD2 + TLR synergy (data not shown). It is tempting to speculate that $1,25 \mathrm{D}$ may differently regulate strong signals induced by combined TLR stimulation and those provided by single TLR ligation. Further experiments are needed to explore this hypothesis.

In Mo-DCs, 1,25D enhanced MDP + LPS + R848 cytokine responses. The differences between PBMCs and Mo-DCs could be caused by dissimilarity in the expression levels of PRR and in signal transduction, different responses to $1,25 \mathrm{D}$, or the presence of multiple cell types in PBMCs. Alternatively, it could reflect the fact that Mo-DCs are specialized proinflammatory dendritic cells more capable of mounting an effective cytokine response against microbes. The identification of the signaling pathways responsible for this difference could not be achieved because of the limited number of cells isolated from patients in this study. However, we observed that $1,25 \mathrm{D}$ resulted in upregulated levels of phosphorylated ERK in PBMCs but not in Mo-DCs. On the other hand, the modulation of I $\mathrm{KB}-\alpha$ expression by $1,25 \mathrm{D}$ was similar in both cell types.

NOD2 mutations are well established as an important genetic risk factor, but are not sufficient to cause CD. It is believed that the presence of other susceptibility alleles in concert with environmental factors such as vD insufficiency can participate in the initiation and perpetuation of $\mathrm{CD}$. Defective innate immunity is suspected to underlie $\mathrm{CD}$ pathogenesis, and 1,25D has the potential to increase response to NOD2 stimulation. Such a therapeutic intervention could be of potential benefit to patients, with the exception of those homozygous for 1007 fs NOD2. However, properly conducted clinical trials would be needed prior to recommending that $\mathrm{vD}$ supplementation become a therapeutic intervention for $\mathrm{CD}$.

\section{METHODS}

Patients, isolation of PBMCs, and generation of Mo-DCs. CD and UC patients were recruited at the IBD clinic at the McGill University Health Center. Healthy controls consisted of age- and gender-matched individuals without immune-mediated gastrointestinal or other autoimmune disorders. Informed consent was obtained as per protocol approved by the McGill University Human Subjects Research Review Committee. Patients were classified as vD sufficient $\left(25(\mathrm{OH}) \mathrm{D}>75 \mathrm{nmoll}^{-1}\right)$, insufficient $(25(\mathrm{OH}) \mathrm{D}<75$ and $\left.>50 \mathrm{nmoll}^{-1}\right)$, or deficient $\left(25(\mathrm{OH}) \mathrm{D}<50 \mathrm{nmoll}^{-1}\right)$. PBMCs were isolated from heparinized venous blood by using density-gradient centrifugation over Ficoll-Paque PLUS (GE Healthcare, Baie d'Urfe, QC, Canada) and plated at a concentration of $3 \times 10^{6} \mathrm{cells} \mathrm{ml}^{-1}$. Monocytes were isolated using CD14 microbeads, MS columns, and the VarioMACS Separation System (Miltenyi Biotec, Auburn, CA). Immature Mo-DCs were generated by culturing isolated monocytes in the presence of IL-4 (500 $\mathrm{U} \mathrm{ml}^{-1}$, Peprotech, Rocky Hill, NJ) and granulocyte macrophage colony-stimulating factor $\left(800 \mathrm{Uml}^{-1}\right.$, Peprotech) for 6 days. On day 3, half of the medium was replaced with fresh culture medium. Day $6 \mathrm{Mo}$-DCs were plated at a concentration of $5 \times 10^{5} \mathrm{cells} \mathrm{ml}^{-1}$. In cases in which insufficient cell numbers were isolated, a second blood draw was organized in order to complete all experimental conditions for all recruited cases.

Cell culture and stimulation. On the basis of results from pilot experiments, PBMCs were incubated with $40 \mathrm{~nm} 1,25-\mathrm{vD}(1,25 \mathrm{D})$ or vehicle for $20 \mathrm{~h}$. The cells were then stimulated with the following PRR agonists for $20 \mathrm{~h}$ : LPS (100 $\mathrm{ng} \mathrm{ml}^{-1}$, from E. coli 026:B6), Pam3CSK4 $\left(2 \mu \mathrm{g} \mathrm{ml}^{-1}\right)$, R848 $\left(2 \mu \mathrm{g} \mathrm{ml}^{-1}\right)$, and MDP $\left(5 \mu \mathrm{g} \mathrm{ml}^{-1}\right)$, all from InvivoGen (San Diego, CA). At the end of each culture period, supernatants were collected and stored at $-70{ }^{\circ} \mathrm{C}$. Vehicle or $1,25 \mathrm{D}$ $(15 \mathrm{nM})$ was added to Mo-DCs in the presence of IL-4 and granulocyte macrophage colony-stimulating factor for $20 \mathrm{~h}$. Cells were stimulated as described above and supernatants were collected after $24 \mathrm{~h}$ for cytokine measurements.

Measurement of cytokines in culture supernatants. Levels of TNF- $\alpha$, IL-10, IL-12/IL-23p40, and IL-12p70 were measured in the supernatants using ELISA kits (R\&D systems, Minneapolis, MN) according to the manufacturer's instructions. IL-23 levels were measured with ELISA kits from eBiosciences (San Diego, CA).

Genotyping. DNA was isolated from whole blood using the Puregene isolation kit (Gentra Systems, Minneapolis, MN) according to the manufacturer's protocol. Genotyping was carried out at the McGill University-Genome Quebec Innovation Center (MUG-QIC) using the sequenom platform as previously described. ${ }^{50}$

Gene expression profiles in THP-1 cells. RNA isolation, cDNA synthesis, and real-time quantitative PCR (RT-qPCR) were performed using standard techniques. The primers used are detailed in Supplementary Table S1.

Statistics. Data were analyzed using GraphPad Prism, version 5.03 (GraphPad Software, La Jolla, CA). All data are presented as median. The statistical significance of the differences induced by $1,25 \mathrm{D}$ was determined using the Wilcoxon matched-pairs signed rank test. The difference between groups was determined using the Mann-Whitney test. The statistical significance of the differences between $\mathrm{CD}$, UC, and control patients was assessed using the Kruskal-Wallis test followed by Dunn's test. Correlation between $1,25 \mathrm{D}$ effect and serum $25(\mathrm{OH}) \mathrm{D}$ levels was tested with the Pearson correlation coefficient. A $P$ value $<0.05$ was considered statistically significant. 
SUPPLEMENTARY MATERIAL is linked to the online version of the paper at http://www.nature.com/mi

\section{ACKNOWLEDGMENTS}

This study was supported by a team grant in autoimmunity from the Canadian Institutes for Health Research (C.P., E.G.S.), and by Canada Research Chairs in Immunoregulation (C.P.), and Immune-Mediated Gastrointestinal Disorders (E.G.S.). Funding was also provided by the Owen Catchpaugh award for innovative research from the McGill University Health Centre Research Foundation. The author thanks Denise Levesque for her expert technical assistance and Melissa Diamond for expertise in ethics and grant administration.

\section{DISCLOSURE}

The authors declared no conflict of interest.

c) 2014 Society for Mucosal Immunology

\section{REFERENCES}

1. Saleh, M. \& Elson, C.O. Experimental inflammatory bowel disease: insights into the host-microbiota dialog. Immunity 34, 293-302 (2011).

2. Kaser, A., Zeissig, S. \& Blumberg, R.S. Inflammatory bowel disease. Annu. Rev. Immunol. 28, 573-621 (2010).

3. Rai, E. \& Wakeland, E.K. Genetic predispositions to autoimmunity - what have we learned? Semin. Immunol. 23, 67-83 (2011).

4. Jostins, L. et al. Host-microbe interactions have shaped the genetic architecture of inflammatory bowel disease. Nature 491, 119-124 (2012).

5. Franke, A. etal. Genome-wide meta-analysis increases to 71 the number of confirmed Crohn's disease susceptibility loci. Nat. Genet. 42, 1118-1125 (2010).

6. Khor, B., Gardet, A. \& Xavier, R.J. Genetics and pathogenesis of inflammatory bowel disease. Nature 474, 307-317 (2011).

7. Hugot, J.P. et al. Association of NOD2 leucine-rich repeat variants with susceptibility to Crohn's disease. Nature 411, 599-603 (2001).

8. Bonen, D.K. et al. Crohn's disease-associated NOD2 variants share a signaling defect in response to lipopolysaccharides and peptidoglycan. Gastroenterology 124, 140-146 (2003).

9. Van Heel, D.A. et al. Muramyl dipeptide and Toll-like receptor sensitivity in NOD2-associated Crohn's disease. Lancet 365, 1794-1796 (2005).

10. Noguchi, E., Homma, Y., Kang, X., Netea, M.G. \& Ma, X. A Crohn's disease-associated NOD2 mutation suppresses transcription of human IL10 by inhibiting activity of the nuclear ribonucleoprotein hnRNP-A1. Nat. Immunol. 10, 471-479 (2009).

11. Voss, E., Wehkamp, J., Wehkamp, K., Stange, E.F., Schroder, J.M. \& Harder, J. NOD2/CARD15 mediates induction of the antimicrobial peptide human beta-defensin-2. J. Biol. Chem. 281, 2005-2011 (2006).

12. Wang, T.T. et al. Direct and indirect induction by 1,25 -dihydroxyvitamin D3 of the NOD2/CARD15-defensin b2 innate immune pathway defective in Crohn Disease. J. Biol. Chem. 285, 2227-2231 (2010).

13. Travassos, L.H. et al. NOD1 and NOD2 direct autophagy by recruiting ATG16L1 to the plasma membrane at the site of bacterial entry. Nat. Immunol. 11, 55-62 (2009).

14. Cooney, R. et al. NOD2 stimulation induces autophagy in dendritic cells influencing bacterial handling and antigen presentation. Nat. Med. 16 90-97 (2010).

15. Homer, C.R., Richmond, A.L., Rebert, N.A., Achkar, J.P. \& McDonald, C. ATG16L1 and NOD2 interact in an autophagy-dependent antibacterial pathway implicated in Crohn's disease pathogenesis. Gastroenterology 139, 1630-1641 (2010).

16. Molodecky, N.A. \& Kaplan, G.C. Environmental risk factors for inflammatory bowel disease. Gastroenterol. Hepatol. 5, 339-346 (2010).

17. Logan, I. \& Bowlus, C.L. The geoepidemiology of autoimmune intestinal diseases. Autoimmun. Rev. 9, A372-A378 (2010).

18. Khalili, H. etal. Geographical variation and incidence of inflammatory bowel disease among US women. Gut 61, 1686-1692 (2012).

19. Garg, M., Lubel, J.S., Sparrow, M.P., Holt, S.G. \& Gibson, P.R. Review article: vitamin $D$ and inflammatory bowel disease - established concepts and future directions. Aliment. Pharmacol. Ther. 36, 324-344 (2012).
20. Pappa, H.M. et al. Vitamin D status in children and young adults with inflammatory bowel disease. Pediatrics 118, 1950-1961 (2006).

21. Ananthakrishnan, A.N. et al. Higher predicted vitamin D status is associated with reduced risk of Crohn's disease. Gastroenterology 142, 482-489 (2012).

22. Nicholson, I., Dalzell, A.M. \& El-Matary, W. Vitamin D as a therapy for colitis: a systematic review. J. Crohn's Colitis 6, 405-411 (2012).

23. Jorgensen, S.P. et al. Clinical trial: vitamin D3 treatment in Crohn's disease - a randomized double-blind placebo-controlled study. Aliment. Pharmacol. Ther. 32, 377-383 (2010).

24. Froicu, M., Weaver, V., Wynn, T.A., McDowell, M.A., Welsh, J.E. \& Cantorna, M.T. A crucial role for the vitamin D receptor in experimental inflammatory bowel diseases. Mol. Endocrinol. 17, 2386-2392 (2003).

25. Liu, N. et al. Altered endocrine and autocrine metabolism of vitamin $D$ in a mouse model of gastrointestinal inflammation. Endocrinology 149, 4799-4808 (2008).

26. Cantorna, M.T., Munsick, C., Berniss, C. \& Mahon, B.D. 1,25-dihydroxycholecalciferol prevents and ameliorates symptoms of experimental murine inflammatory bowel disease. J. Nutr. 130, 2648-2652 (2000).

27. Daniel, C., Sartory, N.A., Zahn, N., Radeke, H. \& Stein, J.M. Immune modulatory treatment of trinitrobenzene sulfonic acid colitis with calcitrol is associated with change of a T Helper (Th) $1 /$ Th17 to a Th2 and Regulatory T cell profile. J. Pharmacol. Exp. Ther. 324, 23-33 (2008).

28. Hewison, M. Vitamin D and the immune system: new perspectives on an old theme. Rheum. Dis. Clin. North Am. 38, 125-139 (2012).

29. Peelen, E. et al. Effects of vitamin D on the peripheral adaptive immune system: a review. Autoimmun. Rev. 10, 733-743 (2011).

30. Gynther, P., Toropainen, S., Matilainen, J.M., Seuter, S., Carlberg, C. \& Väisänen, S. Mechanism of 1 1 ,25-dihydroxyvitamin D(3)-dependent repression of interleukin-12B. Biochim. Biophys. Acta 1813, 810-818 (2011).

31. Lim, W.-C., Hanauer, S.B. \& Li, Y.C. Mechanisms of disease: vitamin D and inflammatory bowel disease. Nat. Rev. Gastroenterol. Hepatol. 2, 308-315 (2005).

32. Smith, A.M. et al. Disordered macrophage cytokine secretion underlies impaired acute inflammation and bacterial clearance in Crohn's disease. J. Exp. Med. 206, 1882-1887 (2009).

33. Seidelin, J.B., Broom, O.J., Olsen, J. \& Nielsen, O.H. Evidence for impaired CARD15 signaling in Crohn's disease without disease linked variants. PLoS One 4, e7794 (2009).

34. Chang, S.H., Chung, Y. \& Dong, C. Vitamin D suppresses Th17 cytokine production by inducing C/EBP homologous protein (CHOP) expression. J. Biol. Chem. 285, 38751-38755 (2010).

35. Joshi, S. et al. 1,25-dihydroxyvitamin $\mathrm{D}(3)$ ameliorates Th17 autoimmunity via transcriptional modulation of interleukin-17A. Mol. Cell. Biol. 31, 3653-3669 (2011).

36. Yuk, J.M. et al. Vitamin D3 induces autophagy in human monocytes/ macrophages via cathelicidin. Cell Host Microbe 6, 231-243 (2009).

37. Schwalfenberg, G.K. Solar radiation and vitamin D: mitigating environmental factors in autoimmune disease. J. Environ. Public Health 2012, ID619381; doi:10.1155/2012/619381 (2012).

38. Antico, A., Tampoia, M., Tozzoli, R. \& Bizzaro, N. Can supplementation with vitamin $\mathrm{D}$ reduce the risk or modify the course of autoimmune diseases? A systematic review of the literature. Autoimmun. Rev. 12, 127-136 (2012).

39. Sadeghi, K. et al. Vitamin D3 down-regulates monocyte TLR expression and triggers hyporesponsiveness to pathogen-associated molecular patterns. Eur. J. Immunol. 36, 361-370 (2006).

40. Khoo, A.L. etal. Regulation of cytokine responses by seasonality of vitamin D status in healthy individuals. Clin. Exp. Immunol. 164, 72-79 (2011).

41. Stio, M. et al. Interaction among vitamin D(3) analogue KH 1060, TNFalpha, and vitamin $\mathrm{D}$ receptor protein in peripheral blood mononuclear cells of inflammatory bowel disease patients. Int. Immunopharmacol. 6 , 1083-1092 (2006).

42. Trinchieri, G. \& Sher, A. Cooperation of Toll-like receptor signals in innate immune defense. Nat. Rev. Immunol. 7, 179-190 (2007).

43. Li, J. et al. Regulation of IL-8 and II-1 beta expression in Crohn's disease associated NOD2/CARD15 mutations. Hum. Mol. Genet. 13, 1715-1725 (2004).

44. van Heel, D.A. et al. Synergy between TLR9 and NOD2 innate immune responses is lost in genetic Crohn's disease. Gut 54, 1553-1557 (2005). 
45. Beynon, V. et al. NOD2/CARD15 genotype influences MDP-induced cytokine release and basal IL-12p40 levels in primary isolated peripheral blood monocytes. Inflamm. Bowel Dis. 14, 1033-1040 (2008).

46. Kramer, M., Netea, M.G., de Jong, D.J., Kullberg, B.J. \& Adema, G.J. Impaired dendritic cell function in Crohn's disease patients with NOD2 3020insC mutation. J. Leukoc. Biol. 79, 860-866 (2006).

47. Yazdanyar, S. \& Weischer, M. Nordestgaard. Genotyping for NOD2 genetic variants and Crohn's disease: a meta-analysis. Clin. Chem. 55, 1950-1957 (2009).
48. Salucci, V. et al. Monocyte-derived dendritic cells from Crohn patients show differential NOD2/CARD15-dependent immune responses to bacteria. Inflamm. Bowel Dis. 14, 812-818 (2008).

49. Peeters, H. et al. CARD15 variants determine a disturbed early response of monocytes to adherent-invasive Escherichia coli strain LF82 in Crohn's disease. Int. J. Immunogenet. 34, 181-191 (2007).

50. Amre, D. et al. Autophagy gene ATG16L1 but not IRGM is associated with Crohn's disease in Canadian children. Inflamm Bowel Dis 15, 501-507 (2009). 